\title{
Incidence of retinal detachment associated with atopic dermatitis in Japan: review of cases from I 992 to 201 I
}

This article was published in the following Dove Press journal:

Clinical Ophthalmology

23 June 2015

Number of times this article has been viewed

\section{Mikio Sasoh ${ }^{1,2}$ \\ Hitoshi Mizutani ${ }^{3}$ \\ Hisashi Matsubara' \\ Motoyasu Furuta ${ }^{4}$ \\ Yoshitsugu Matsui' \\ Kei-ichi Yamanaka ${ }^{3}$ \\ Mineo Kondo'}

'Department of Ophthalmology, Graduate School of Medicine, Mie University, Tsu, ${ }^{2}$ Local Independent Administrative Institution, Mie Prefectural General Medical Center, Yokkaichi, ${ }^{3}$ Department of Dermatology, Graduate School of Medicine, Mie University, Tsu, ${ }^{4}$ Japanese Red Cross Ise Hospital, Ise, Mie, Japan
Correspondence: Mikio Sasoh

Department of Ophthalmology, Graduate School of Medicine, Mie University, 2-174 Edobashi, Tsu, Mie 514-8507, Japan

Tel $+8 I 5923$ I 5027

Fax +81592313036

Email sasoh8521@yahoo.co.jp
Purpose: The present study aims to investigate the number and characteristics of retinal detachment with atopic dermatitis (AD) in these 20 years, and the number of the first visit $A D$ outpatients in almost the same period.

Methods: A retrospective review of 101 consecutive surgically treated retinal detachments with AD patients from 1992 to 2011 was conducted. Retinal detachments were divided into two groups: eyes operated on from 1992 to 2001 (former AD group, $n=63$ ) and eyes operated on from 2002 to 2011 (recent AD group, $n=38$ ). We also reviewed the records of the first visit AD outpatients from 1993 to 2011 except 1998.

Results: The percentage of bilateral detachment was significantly higher in the former AD group (14/63) than that in the recent AD group (0/38) $(P=0.0002)$. In addition, patients in the recent $\mathrm{AD}$ group were significantly older than those in the former $\mathrm{AD}$ group $(P=0.0084)$. The annual cases with non-AD retinal detachment remained invariant for 20 years. The ratio of the retinal detachment with $\mathrm{AD}$ for the total retinal detachment was significantly lower in the recent (38/847) AD group than that in the former (63/796) AD group ( $P=0.0038)$. The number of the first visit AD outpatients linearly decreased in these 19 years (153 cases in 1993 and 65 cases in 2011).

Conclusion: Our study indicates an apparent decrease in retinal detachment with AD in the recent 10 years, and might suggest the importance of dermatitis control for prevention of retinal detachment with AD.

Keywords: atopic dermatitis, incidence, ocular complication, retinal detachment

\section{Introduction}

Ocular complications of atopic dermatitis (AD) include blepharitis, keratoconjunctivitis, keratoconus, iritis, cataract, and retinal detachment. ${ }^{1}$ Among these, retinal detachment is the severest complication that affects the younger population and significantly impairs their quality of life. Previous single center ${ }^{2}$ or multicenter ${ }^{3,4}$ retrospective studies declared the presence of a large number of retinal detachment patients with $\mathrm{AD}$ and increase in retinal detachment patients with AD until 1993. ${ }^{2,4}$ The increase in retinal detachment patients with $\mathrm{AD}$ was attributed to the marked increase in severely involved AD patients in late 1980s.$^{5-7}$ However, no further detailed studies for retinal detachment with AD has been reported after 2000s. Thus, the current status of retinal detachment with AD is unknown.

The prevalence of adolescent AD markedly increased from 1967 to 1996 in Japan. ${ }^{8}$ After 2000, AD therapies improved by introduction of new therapeutic tools including the topical and systemic calcineurin inhibitors, and new therapeutic strategy: proactive 
therapies with topical calcineurin inhibitors and/or corticosteroids. Improvement of AD therapies decreased the prevalence of AD in 2006 compared with that in $1993 .{ }^{9}$ In Japan, topical tacrolimus therapy is approved as an effective and well-tolerated treatment option for patients with AD. ${ }^{10}$ These recent changes in AD therapies might influence the prevalence of retinal detachment with AD. Therefore, ophthalmologists as well as dermatologists have great interest to the new trend in retinal detachment associated with AD. Herein, we report changes in surgically treated retinal detachment in Mie University Hospital, Mie, Japan, for these 20 years.

\section{Patients and methods}

The present study adhered to the tenets of the Declaration of Helsinki, and was approved by the Institutional Review Board of the Mie University Graduate School of Medicine (\#2619). We retrospectively reviewed the records of 1,533 consecutive patients including AD patients with rhegmatogenous retinal detachment, who underwent retinal detachment surgery at Mie University Hospital during a 20-year period that ranged from January 1992 to December 2011. The data set examined included patient's age, sex, affected eye, preoperative and postoperative best-corrected visual acuity, type of breaks, preoperative lens status, macula and the ciliary epithelium status, and the surgical results. We also reviewed the records of new AD outpatients consulted to the dermatology clinic at Mie University Hospital from 1993 to 2011 except 1998.

Cases with retinal detachments were divided into two groups: retinal detachments with $\mathrm{AD}$ (AD group) and without AD (non-AD group). Subsequently, the AD group was divided into two subgroups: patients group surgically treated former 10 years of the study period: from 1992 to 2001 (former AD group) and another group treated in recent 10 years from 2002 to 2011 (recent AD group). We also analyzed the annual changes in the number of retinal detachments with $\mathrm{AD}$ and the total retinal detachments (Figure $1 \mathrm{~A}$ and $\mathrm{B}$ ). The number of the first visit AD outpatients to the dermatology clinic was also described in Figure 1C.

Data were analyzed between the AD and non-AD groups, or between the former $\mathrm{AD}$ and recent $\mathrm{AD}$ groups. With the exception of patient's age, all preoperative characteristics and reattachment rate were statistically analyzed using either a chi-square test or a Fisher's exact probability test. A MannWhitney $U$-test was used for analyses of the patient's age and visual acuity. Differences were considered as significant when the probability of their occurrence by chance was less than $5 \%$.

\section{Results \\ Difference between the AD group and the non-AD group}

The characteristic differences between the two groups (AD group and non-AD group) are as shown in Table 1 . The ratio of the bilateral detachment cases was significantly higher in the $\mathrm{AD}$ group than that in the non-AD group $(P=0.0011)$. Patients in the $\mathrm{AD}$ group were significantly younger than those in the non-AD group $(P<0.0001)$. The breaks were more frequently observed in the peripheral ocular fundus in the AD group compared with the non-AD group $(P<0.0001)$. A greater number of eyes had macular detachments before surgery in the non-AD vs the AD group ( $P=0.0052)$. Ciliary epithelium detachment developed more frequently in the $\mathrm{AD}$ vs the non-AD group $(P<0.0001)$. Although the reattachment rate after the first surgery in the $\mathrm{AD}$ group $(76.2 \%, 77 / 101)$ appeared to be a little worse than that in the non-AD group $(81.5 \%, 1,257 / 1,542)$, the difference in the rate of anatomical success was not statistically significant $(P=0.2364)$. Final reattachment rate in the AD group (98.0\%, 99/101) was almost the same as that in the non-AD group $(98.1 \%, 1,513 / 1,542)(P=0.7157)$. In spite of no significant difference in the preoperative best-corrected visual acuity between the two groups, the postoperative best-corrected visual acuity was significantly better in the $\mathrm{AD}$ group compared with the non-AD group ( $P=0.0002$; Table 2$)$. In the $\mathrm{AD}$ group, 51 eyes had cataract before retinal detachment surgery, and the 23 eyes out of the 51 eyes underwent cataract surgery during retinal detachment surgery.

\section{Difference between the former and recent $A D$ groups}

The characteristic differences between the two groups (former $\mathrm{AD}$ group and recent $\mathrm{AD}$ group) are as shown in Table 3. The bilateral detachment percentage was significantly higher in the former $\mathrm{AD}$ group than that in the recent AD group $(P=0.0002)$. Patients in the recent $\mathrm{AD}$ group were significantly older than those in the former AD group $(P=0.0084)$. No significant difference was identified between the groups for the type of break, lens status, macular status, or rate of ciliary epithelial detachment. The ratio of the retinal detachment with $\mathrm{AD}$ for the total retinal detachment was significantly lower in the recent $\mathrm{AD}$ group compared with the former $\operatorname{AD}$ group $(P=0.0038)$. Although the reattachment rate after the first surgery in the former $\mathrm{AD}$ group $(73.0 \%$, $46 / 63)$ appeared to be a little worse than that in the recent AD group $(81.6 \%, 31 / 38)$, the difference in the rate of anatomical success was not statistically significant $(P=0.4589)$. 

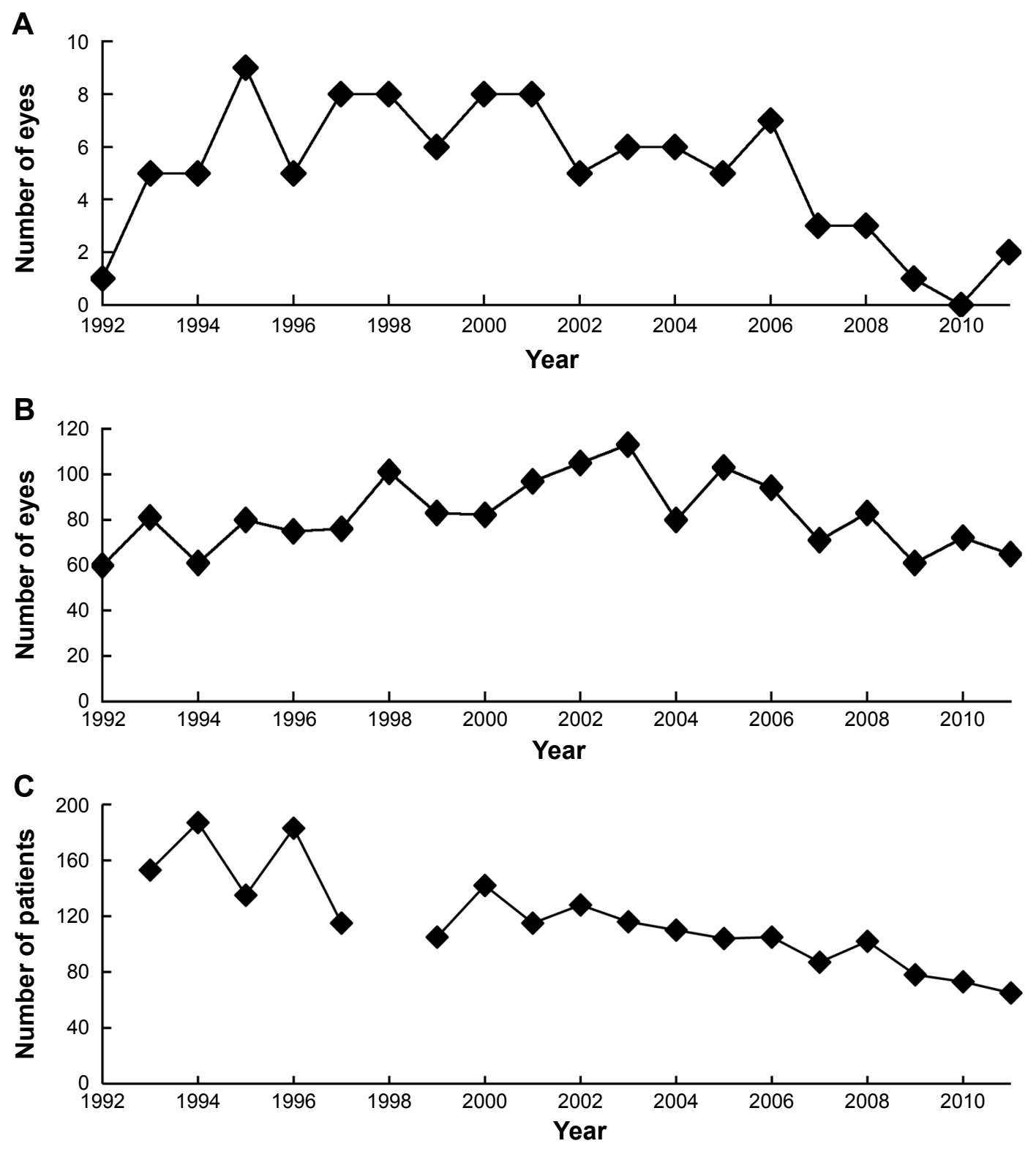

Figure I Annual changes in the number of surgically treated retinal detachments, and atopic dermatitis patients.

Notes: (A) Number of retinal detachments with atopic dermatitis each year. (B) Number of total retinal detachments each year. (C) Annual changes in atopic dermatitis patients. The number of new atopic dermatitis outpatients seen in the Department of Dermatology, Mie University, Graduate School of Medicine each year. Data for the year 1998 were not available.

Final reattachment rate in the former $\mathrm{AD}$ group $(98.4 \%, 62 / 63)$ was almost the same as that in the recent $\mathrm{AD}$ group $(97.4 \%$, 37/38) $(P>0.9999)$. The functional outcome, including preoperative best-corrected visual acuity and postoperative best-corrected visual acuity, showed no statistical difference between the former and recent AD groups (Table 4).

\section{Annual changes in the number of surgically treated retinal detachments with $A D$}

The number of retinal detachments with $\mathrm{AD}$ and the total number of retinal detachments are described in Figure 1. No apparent change was observed in the annual number of the total retinal detachments for these 20 years (Figure 1B). In contrast, the number of retinal detachments with AD rapidly increased from 1992 to 1995 and continued until 2001 (Figure 1A). After 2001, it decreased gradually. Especially, no retinal detachment with AD was treated in 2010. Because of no apparent change in total retinal detachments, the rate of retinal detachment with AD in the total retinal detachment also increased from 1992, and it tapered in 2000s (data not shown).

\section{First visit $A D$ patients}

The number of new AD outpatients in our dermatology clinic linearly decreased in these 19 years (153 cases in 1993 and 
Table I Preoperative characteristics of I,643 eyes

\begin{tabular}{|c|c|c|c|}
\hline & AD group & Non-AD group & $P$-value \\
\hline Total number & I0I eyes, 87 patients & I,542 eyes, I,446 patients & \\
\hline Unilateral:Bilateral (patient) & $73: 14$ & I,350:96 & 0.0011 \\
\hline Male:Female (patient) & $56: 31$ & $860: 586$ & 0.4287 \\
\hline Side, Right:Left (eye) & $59: 42$ & $851: 691$ & 0.5968 \\
\hline Age, year, range (mean) & $12-57(22.9)$ & $6-9 \mid(51.3)$ & $<0.0001$ \\
\hline \multicolumn{4}{|l|}{ Retinal or ciliary break } \\
\hline Pars plicata & $30(17.9 \%)$ & $2(0.1 \%)$ & \\
\hline Pars plana & 29 (I7.4\%) & 17 (0.7\%) & \\
\hline Ora & $49(29.3 \%)$ & 81 (3.2\%) & \\
\hline Tear & 32 (19.2\%) & I,360 (53.4\%) & \\
\hline Hole & 27 (16.2\%) & $956(37.5 \%)$ & \\
\hline Macular hole & $0(0 \%)$ & $|3|(5.1 \%)$ & $<0.000$ I \\
\hline Macular, Off:On (eye) & $37: 64$ & $786: 756$ & 0.0052 \\
\hline CD (+):CD (-) (eye) & $85: 16$ & $76: 1,466$ & $<0.000$ I \\
\hline
\end{tabular}

Abbreviations: $A D$, atopic dermatitis; $C D$, detachment of ciliary epithelium.

65 cases in 2011, Figure 1C). Their average age gradually increased during the study period (mean age of 19.41 years old in 1993 and 24.94 years old in 2011).

\section{Discussion}

Retinal detachment is a serious eye disease that impairs quality of life of the patients, and a significant increase in cases with retinal detachment in AD patients has been reported in 1990s. By advance in understanding of AD pathogenesis and improvement of therapies, prognosis of the severely involved AD patients has been improved. To know recent changes in prevalence of retinal detachment including with/without AD, we investigated the surgically treated patients with retinal detachment during these 20 years in our hospital.

Reported retinal detachment cases associated with AD are characterized with common bilateral involvement and development in the younger age compared with the cases without AD. The causative break is frequently located in the peripheral ocular fundus, with the ciliary epithelium found to be detached in many of the cases. ${ }^{2-4,11}$ These characteristics are also found in the present study. In addition, the present results also demonstrated that postoperative visual functions in $\mathrm{AD}$ patients were better than those found in the non-AD patients (Table 2 ). These findings might be resulted from younger age and higher ratio of preoperative macular attachment in the affected eyes in AD patients compared with non-AD patients. In the present data, there was no significant difference in the preoperative best-corrected visual acuity between the $\mathrm{AD}$ and non-AD groups in spite of higher ratio of preoperative macular attachment in the AD group. The discrepancy might be resulted from high ratio of preoperative cataract in $\mathrm{AD}$ patients.

In the present study, the number of retinal detachments with AD markedly varied in these 20 years. It rapidly increased from 1992 to 1993, remained same levels until 2001, and gradually decreased year by year after 2001. The annual number of the total retinal detachments showed neither particular increase nor decrease for 20 years with minimum yearly changes. Then, the rate of retinal detachments with AD in the total retinal detachments was evaluated. It increased in early 1990s and decreased in 2000s as well as the number of retinal detachments with AD. Thus, we concluded that the prevalence of retinal detachments with AD rapidly increased in early 1990s and decreased in 2000s. Cases with bilateral retinal detachment decreased in the recent 10 years, and this contributed to the decrease in the number of retinal detachments with $\mathrm{AD}$ in part. It is easy to suspect that bilateral involvement has resulted from severe AD inflammation on the face. Unfortunately, the disease activities of $\mathrm{AD}$ in these surgically treated cases have not been fully recorded.

Table 2 Visual acuity before and after surgery: the comparison between AD and non-AD groups

\begin{tabular}{|c|c|c|c|c|c|c|}
\hline & \multicolumn{3}{|c|}{ Preoperative visual acuity } & \multicolumn{3}{|c|}{ Postoperative visual acuity } \\
\hline & AD group (\%) & Non-AD group (\%) & $P$-value & AD group (\%) & Non-AD group (\%) & $P$-value \\
\hline $20 / 15$ to $20 / 25$ & 39 (39.8\%) & $555(37.4 \%)$ & & $62(63.3 \%)$ & $703(47.4 \%)$ & \\
\hline $20 / 30$ to $20 / 50$ & $2 \mathrm{I}(2 \mathrm{I} .4 \%)$ & $224(15.1 \%)$ & & $20(20.4 \%)$ & $337(22.7 \%)$ & \\
\hline $20 / 60$ to $20 / 200$ & $18(18.4 \%)$ & $226(15.3 \%)$ & & $12(12.2 \%)$ & 294 (19.8\%) & \\
\hline Less than $20 / 200$ & 20 (20.4\%) & 478 (32.2\%) & 0.1498 & $4(4.1 \%)$ & 149 (10.1\%) & 0.0002 \\
\hline
\end{tabular}

Abbreviation: $A D$, atopic dermatitis. 
Table 3 Preoperative characteristics of $10 \mathrm{I}$ eyes

\begin{tabular}{llll}
\hline & Former AD group & Recent AD group & P-value \\
\hline Total number & 63 eyes, 49 patients & 38 eyes, 38 patients \\
Unilateral:Bilateral (patient) & $35: 14$ & $38: 0$ & 0.0002 \\
Male:Female (patient) & $28: 21$ & $28: 10$ & 0.1692 \\
Side, Right:Left (eye) & $37: 26$ & $22: 16$ & $12-57(25.6)$ \\
Age, year, range (mean) & $12-41(21.2)$ & & 0.9342 \\
Retinal or ciliary break & & $8(13.3 \%)$ & 0.0084 \\
Pars plicata & $22(21.0 \%)$ & $10(16.7 \%)$ \\
Pars plana & $19(18.1 \%)$ & $15(25.0 \%)$ \\
Ora & $34(32.4 \%)$ & $14(23.3 \%)$ \\
Tear & $18(17.1 \%)$ & $13(21.7 \%)$ \\
Hole & $12(11.4 \%)$ & $7(18.4 \%)$ \\
Lens status & & $18(47.4 \%)$ \\
Cataract $(-)$ & $15(23.8 \%)$ & $4(10.5 \%)$ \\
Cataract (+) & $33(52.4 \%)$ & $9(23.7 \%)$ \\
Aphakia & $10(15.9 \%)$ & $13: 25$ \\
Pseudophakia & $5(7.9 \%)$ & $32: 6$ \\
Macular, Off:On (eye) & $24: 39$ & $38: 847$ \\
CD (+):CD (-) (eye) & $53: 10$ &
\end{tabular}

Abbreviations: $A D$, atopic dermatitis; $C D$, detachment of ciliary epithelium; $R D$, retinal detachment.

Therefore, we cannot draw any definitive conclusions about this issue.

A decrease in $\mathrm{AD}$ patients is a suspicious factor for the noted decreases in retinal detachment with $\mathrm{AD}$. In our present data as well as in a previous study, ${ }^{9}$ new AD outpatients in our dermatology clinic gradually decreased (Figure 1C). It is also noteworthy that these $\mathrm{AD}$ outpatients were referred to our clinic mainly because of uncontrolled AD. In addition, Mie University Hospital is the only core hospital in Mie prefecture, and most refractory cases in this area have been referred to Mie University Hospital in these 20 years. Therefore, we think that the present data indicate gradual decrease in uncontrolled AD patients in Mie prefecture. Topical corticosteroid therapy is a globally accepted standard therapy. However, "steroid phobia in AD patients" prevented control of the dermatitis with insufficient therapy. Thus, the long-lasting dermatitis results in resistant and uncontrolled AD. ${ }^{9}$ Unfortunately, patients with steroid phobia significantly increased from early 1990s as a social phenomenon in Japan.
Around 2000, the Japanese Dermatological Association declared the definition of $\mathrm{AD}$, guideline for $\mathrm{AD}$ diagnosis and treatment and standard therapy campaign. ${ }^{12}$ Simultaneously, topical calcineurin inhibitor tacrolimus has been introduced in Japan as a new effective non-steroid and less-rosacea forming topical therapy in the end of $1999 .{ }^{13}$ Additionally, a recently introduced new therapeutic strategy "proactive therapy" characterized with intermittent topical corticosteroid and/or calcineurin inhibitors associated with good skin care successfully controlled the relapse of AD. ${ }^{14,15}$ In 2008, systemic cyclosporine was approved for AD in Japan, and it has made resistant $\mathrm{AD}$ cases more controllable. ${ }^{16}$ The decrease in retinal detachment with $\mathrm{AD}$ after 2001 and rapid fall off after 2008 might have relations to these new therapies in part. From these results, frequent rubbing of the eyes in the setting of $\mathrm{AD}$ might be one of the causative factors explaining a higher incidence of retinal detachment in AD patients.

Another striking change noted during the 20-year observation period was the change in age for retinal detachment

Table 4 Visual acuity before and after surgery: the comparison between former and recent AD groups

\begin{tabular}{|c|c|c|c|c|c|c|}
\hline & \multicolumn{3}{|c|}{ Preoperative visual acuity } & \multicolumn{3}{|c|}{ Postoperative visual acuity } \\
\hline & $\begin{array}{l}\text { Former AD } \\
\text { group (\%) }\end{array}$ & $\begin{array}{l}\text { Recent AD } \\
\text { group (\%) }\end{array}$ & $P$-value & $\begin{array}{l}\text { Former AD } \\
\text { group (\%) }\end{array}$ & $\begin{array}{l}\text { Recent AD } \\
\text { group (\%) }\end{array}$ & $P$-value \\
\hline $20 / 15$ to $20 / 25$ & $22(36.1 \%)$ & $17(46.0 \%)$ & & $40(65.6 \%)$ & $22(59.5 \%)$ & \\
\hline $20 / 30$ to $20 / 50$ & $13(21.3 \%)$ & $8(21.6 \%)$ & & $10(16.4 \%)$ & $10(27.0 \%)$ & \\
\hline $20 / 60$ to $20 / 20$ & $10(16.4 \%)$ & $8(21.6 \%)$ & & $7(11.5 \%)$ & $5(13.5 \%)$ & \\
\hline Less than $20 / 200$ & $16(26.2 \%)$ & $4(10.8 \%)$ & 0.1952 & $4(6.5 \%)$ & $0(0.0 \%)$ & 0.8386 \\
\hline
\end{tabular}

Abbreviation: $A D$, atopic dermatitis. 
with AD. During the recent 10 years, patients were found to be significantly older than those observed during the former 10 years (Table 3 ). Both the previous study ${ }^{9}$ and our current data for the new AD outpatients in the dermatology clinic have shown that the age of AD patients has become older year by year. We speculate that there has been an increased onset of AD in older subjects, with this subsequently leading to the onset of retinal detachment with AD.

The present data for retinal detachment with AD during the 20-year observation period revealed no particular changes in ophthalmological manifestations of the affected eye itself, including for the type of breaks, lens status, macular status, or ciliary epithelial detachment. The present study failed to detect any improvement in the anatomical or functional results of the retinal detachment surgeries in these 20 -year periods. We hope that the recent refinements in the vitrectomy system will contribute to the surgical results in future.

\section{Conclusion}

The present study revealed increased retinal detachment with AD in 1990s followed by a gradual decrease in 2000s. Various factors including standard therapy for AD campaign, proactive therapy, and topical and systemic calcineurin inhibitor might contribute to good control of AD disease activities and consequent decrease in retinal detachment with AD. It is clear that further studies for ophthalmological and dermatological analysis for retinal detachment with $\mathrm{AD}$ in a larger scale are required.

\section{Author contributions}

All authors contributed toward data analysis, drafting and revising the paper and agree to be accountable for all aspects of the work.

\section{Disclosure}

Hitoshi Mizutani has received research support and consultant honoraria from Novartis, Astellas, and Maruho, and receives grants (24591647) for scientific research from the Ministry of Education, Culture, Sports, Science and Technology,
Japan. The funders had no role in study design, data collection and analysis, decision to publish, or preparation of the manuscript. The authors declare that there is no conflicts of interest regarding the publication of this paper.

\section{References}

1. Rich LF, Hanifin JM. Ocular complication of atopic dermatitis and other eczemas. Int Ophthalmol Clin. 1985;25(1):61-76.

2. Takahashi M, Suzuma K, Inaba I, Ogura Y, Yoneda K, Okamoto H. Retinal detachment associated with atopic dermatitis. Br J Ophthalmol. 1996;80(1):54-57.

3. Azuma N, Hida T, Katsura H, Takeuchi S, Danjo S, Tano Y. Retrospective survey of surgical outcomes on rhegmatogenous retinal detachments associated with atopic dermatitis. Arch Ophthalmol. 1996;114(3): $281-285$.

4. Hida T, Tano Y, Okinami S, Ogino N, Inoue M. Multicenter retrospective study of retinal detachment associated with atopic dermatitis. Jpn J Ophthalmol. 2000;44(4):407-418.

5. Nishioka K. Adult type of atopic dermatitis. Rinsho Derma. 1991;33(3): 413-418.

6. Levy RM, Gelfand JM, Yan AC. The epidemiology of atopic dermatitis. Clin Dermatol. 2003;21(2):109-115.

7. Mohammedamin RS, van der Wouden JC, Koning S, et al. Increasing incidence of skin disorders in children? A comparison between 1987 and 2001. BMC Dermatol. 2006;6:4. doi:10.1186/1471-5945-6-4.

8. Furue M, Chiba T, Takeuchi S. Current status of atopic dermatitis in Japan. Asia Pac Allergy. 2011;1(2):64-72.

9. Yura A, Kouda K, Iki M, Shimizu T. Trends of allergic symptoms in school children: large-scale long-term consecutive cross-sectional studies in Osaka Prefecture, Japan. Pediatr Allergy Immunol. 2011;22(6): 631-637.

10. Kim KH, Kono T. Overview of efficacy and safety of tacrolimus ointment in patients with atopic dermatitis in Asia and other areas. Int J Dermatol. 2011;50(9):1153-1161.

11. Yoshida S, Sasoh M, Arima M, Uji Y. Ultrasound biomicroscopic view of detachment of the ciliary epithelium in retinal detachment with atopic dermatitis. Ophthalmology. 1997;104(2):283-287.

12. Saeki H, Furue M, Furukawa F, et al. Guidelines for management of atopic dermatitis. J Dermatol. 2009;36(10):563-577.

13. Kawashima M. QOL Research Forum for Patients with Atopic Dermatitis. Quality of life in patients with atopic dermatitis: impact of tacrolimus ointment. Int J Dermatol. 2006;45(6):731-736.

14. Wollenberg A, Reitamo S, Girolomoni G, et al. Proactive treatment of atopic dermatitis in adults with $0.1 \%$ tacrolimus ointment. Allergy. 2008;63(7):742-750.

15. Fukuie T, Nomura I, Horimukai K, et al. Proactive treatment appears to decrease serum immunoglobulin-E levels in patients with severe atopic dermatitis. Br J Dermatol. 2010;163(5):1127-1129.

16. Nakamura S, Takeda K, Hashimoto Y, et al. Favorable clinical response by pre-prandial administration of low-dose ciclosporin to severe adult atopic dermatitis. J Dermatolog Treat. 2012;23(2):112-115.
Clinical Ophthalmology

\section{Publish your work in this journal}

Clinical Ophthalmology is an international, peer-reviewed journal covering all subspecialties within ophthalmology. Key topics include: Optometry; Visual science; Pharmacology and drug therapy in eye diseases; Basic Sciences; Primary and Secondary eye care; Patient Safety and Quality of Care Improvements. This journal is indexed on Submit your manuscript here: http://www.dovepress.com/clinical-ophthalmology-journal

\section{Dovepress}

PubMed Central and CAS, and is the official journal of The Society of Clinical Ophthalmology (SCO). The manuscript management system is completely online and includes a very quick and fair peer-review system, which is all easy to use. Visit http://www.dovepress.com/ testimonials.php to read real quotes from published authors. 